\title{
De l'approche par objectifs à l'approche par compétences. Faut-il jeter le bébé avec l'eau du bain ?
}

\author{
From objective-based to competency-based approach. \\ Should we have to throw out the baby with the bathwater?
}

Si l'on adopte une perspective nominaliste pour examiner l'évolution des concepts qui nous permettent d'imaginer nos pratiques pédagogiques, il semble bien que celui de compétence soit sur le point de supplanter celui d'objectif. Cependant, sa fortune actuelle ne garantit pas une compréhension explicite de ses prémisses épistémologiques, ni même de ses racines étymologiques - du latin cum petere, se diriger vers un même point-. Les approches développées autour du concept de compétence sont en effet polysémiques et plurielles. Comme celles initiées à partir du concept d'objectif, elles ont été successivement influencées par plusieurs courants théoriques, ce qui rend leur abord parfois difficile. Les travaux inspirés par ces nouvelles orientations sont, en outre, surtout disponibles en langue anglaise et certaines traductions françaises, quand elles existent, sont parfois équivoques. Il faut donc saluer les deux contributions de ce numéro ${ }^{l, 2}$, qui apportent des clarifications bienvenues pour comprendre les débats qui entourent l'émergence du concept de compétence en éducation des professionnels de santé.

La notion de compétence concerne en fait trois champs. Le premier recouvre ce quil est aujourd'hui convenu de dénommer " approche pédagogique par compétences". Nguyen et Blais ${ }^{1}$ exposent comment ce cadre conceptuel a émergé pour tenter de dépasser certaines limites reconnues aux développements opérationnels de l'" approche par objectifs". Ils rappellent les influences dont se sont nourris ces deux courants, soulignent leurs contributions respectives mais aussi leurs limites ou difficultés, en montrant comment ils se sont élaborés à la fois en rupture et en continuité. Le deuxième champ est celui de la gestion des ressources humaines dans la société, dans les institutions et dans les milieux professionnels. C'est celui qu'abordent Carré et Gagnayre ${ }^{2}$, autour de la notion de bilan de compétences, en explicitant les convergences mais aussi les différences de cette démarche avec les bilans d'évaluation, les bilans d'orientation, la validation des acquis professionnels ou autres méthodes utilisables pour fournir aux professionnels une rétroaction concernant leur parcours. A l'interface des deux champs précédents, et comme l'évoquent les deux articles, celui de l'evaluation est central et l'on assigne désormais au concept de compétence une finalité fortement structurante à l'égard des activités d'évaluation des apprentissages, des enseignements et des dispositifs de formation ${ }^{3}$.

On peut déplorer que les champs sémantiques et lexicaux qui accompagnent cette évolution soient encore imparfaitement stabilisés. Dans la littérature anglo-saxone, le terme evaluation tend désormais à se restreindre à l'évaluation des programmes et il est substitué par celui d'assessment pour l'evaluation des apprentissages.'. Par ailleurs, le concept d'outcome est mis en correspondance, pour l'en rapprocher, avec celui de compétence (competency) ${ }^{3}$. Dans ce sens, il est employé pour désigner les finalités visées par les programmes, formulées en termes de conséquences attendues et évaluables de l'ensemble des activités d'enseignement et d'apprentissage ; il englobe donc implicitement le concept de compétence, comme résultat délibérément visé à l'issue de certains programmes. Mais le même terme est également mis en correspondance, cette fois pour l'en distinguer, avec celui d'objectif. Bien que les deux locutions soient souvent employées comme synonymes, la notion de learning outcome tend à remplacer celle de learning objective, pour élargir le concept d'objectif au-delà des seuls résultats observables, en intégrant à fois le processus et le produit dans la définition des apprentissages visés ${ }^{3}$. Les expressions dérivées sont nombreuses (competency-based education, comptetencybased curriculum, competency-based assessment, learning competency, outcome-based education, outcome-based assessment, etc.) et les usages respectifs pas toujours faits avec le discernement souhaitable. L'ensemble peut sembler souvent fort déroutant pour un lecteur francophone.

Il n'empêche. Malgré ce foisonnement et ces ambiguités terminologiques, la cause paraît désormais entendue. Après un règne presque sans partage de l'approche par objectifs, c'est dorénavant une nouvelle injonction, celle de l'approche par compétences, qui nous est formulée. Il est dès lors nécessaire d'en examiner loyalement les enjeux.

Avec éloquence, plusieurs auteurs jouissant d'un grand crédit dans le milieu de l'éducation médicale nous ont récemment invité à résister au séduisant attrait du concept de compétences. Grant ${ }^{5}$ perçoit, entre autres, le risque d'une instrumentalisation de l'approche par compétences aux fins d'une tentative de mise sous contrôle socio-économique et politique des professions de santé. On peut à juste titre être réservé à l'égard de ce quilly a de normatif et de prescriptif derrière certains "référentiels de compétences " promus, ici ou là, sous la pression insistante des pouvoirs publics ou d'agences gouvernementales. La transposition imprudente de principes managériaux aux champs de la santé et de l'éducation peut en effet conduire à des applications réductrices du concept de démarche qualité. Lorsque celle-ci est comprise comme une conformité des pratiques par rapport à 


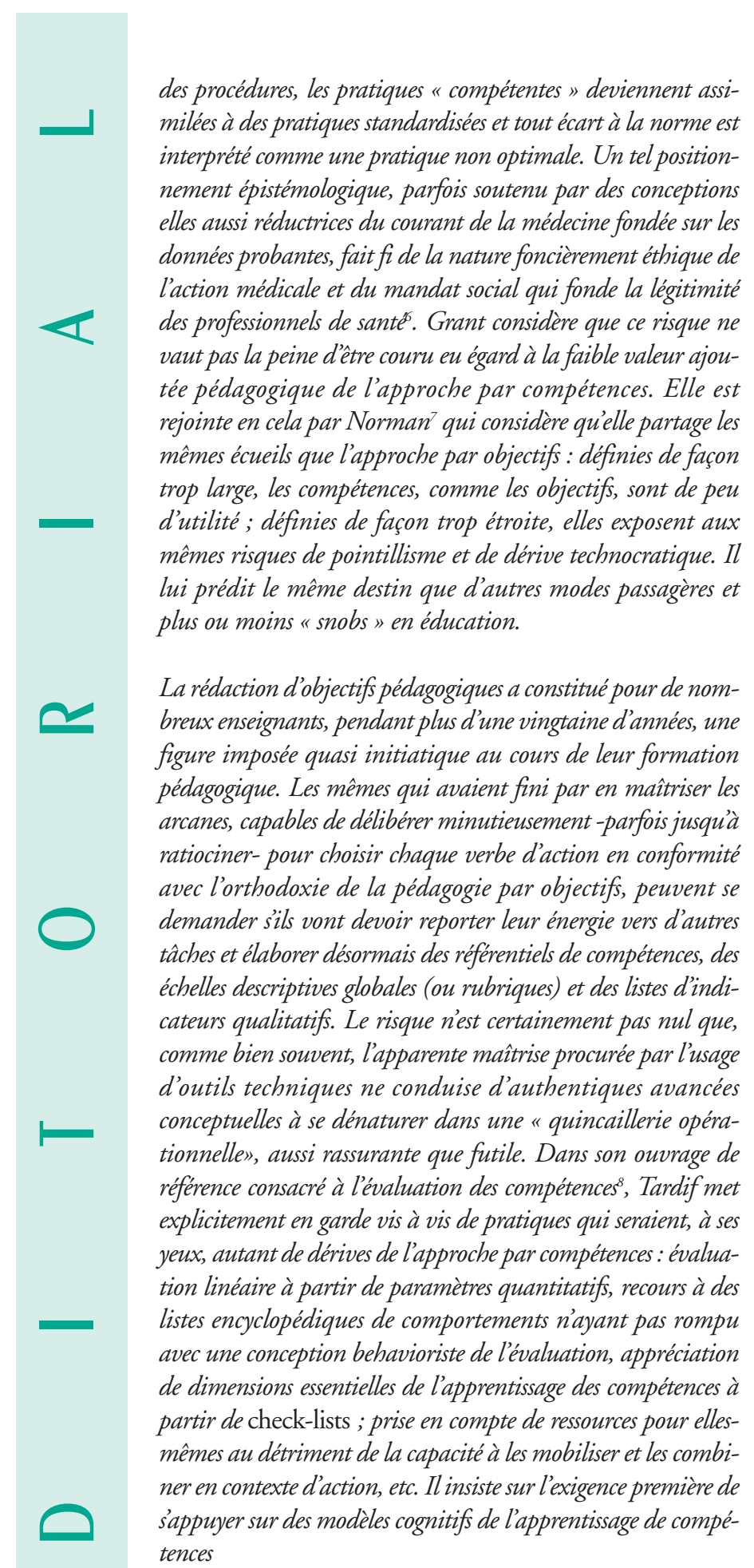

Il est donc essentiel d'appréhender le courant de l'approche par compétences pour ce quill est et ne pas confondre les buts et les moyens. En tant que nouveau paradigme, articulant les apports de plusieurs théories et connaissances issues de la recherche mais aussi des convictions non encore démontrées ainsi que des valeurs partagées, il fournit à la communauté de chercheurs et de praticiens qui sy reconnaissent un cadre conceptuel pour formuler les problèmes auxquels ils sont confrontés et élaborer des hypothèses de solutions. Ni dogme, ni panacée pédagogique, il permet au contraire de cultiver le doute méthodique à l'égard de nos pratiques pédagogiques. En tant que tel, il constitue cependant une rupture dont il faut prendre toute la mesure. La démarche proposée est exigeante et implique notamment, si l'on sy engage, d'accepter un pouvoir de gérance des compétences sur les disciplines et de sinscrire dans une nouvelle culture de collaboration et d'interdépendance professionnelles. Il ne suffira pas d'un travail cosmétique visant à habiller les objectifs en compétences pour rendre plus pertinentes nos actions pédagogiques, pas plus qu'il n'avait suffi, à l'époque, de travestir les contenus disciplinaires en listes d'objectifs. C'est à ce prix seulement que, malgré les légitimes interrogations quelle provoque et en dépit des usages galvaudés qu'elle ne manquera pas de susciter, la notion de compétence pourra savérer extrêmement féconde pour tous les éducateurs impliqués dans la formation des professionnels de santé.

Jean JOUQUAN mailto : jean.jouquan@chu-brest.fr

\section{Références}

1. Nguyen D-Q, Blais J-G. Approche par objectifs ou approche par compétences? Repères conceptuels et implications pour les activités d'enseignement, d'apprentissage et d'évaluation au cours de la formation clinique. Pédagogie Médicale 2007;8:232-51.

2. Carré D, Gagnayre R. Le bilan de compétences : une multiplicité d'approches conceptuelles et de définition au travers de travaux français. Pédagogie Médicale 2007;8:224-31.

3. Anderson HM, Moore DL, Anaya G, Bird E. Student learning outcomes assessment: a component of program assessment. Am J Pharm Educ 2005;69:256-68.

4. Scallon G. L'évaluation des apprentissages dans une approche par compétences. Québec : Editions du renouveau pédagogique, 2004.

5. Grant J. The incapaciting effects of competence : a critique. Adv Health Sci Educ Theory Pract 1999;4:271-7.

6. Jouquan J. L'évaluation des pratiques confrontées à l'exigence de la preuve scientifique en medicine clinique. Mt Thérapeutique 2003;9:93-100.

7. Norman G. Outcomes, objectives, and the seductive appeal of simple solutions. Adv Health Sci Educ Theory Pract $2006 ; 11$ :217-20.

8. Tardif J. L'évaluation des compétences. Documenter le parcours de développement Montréal (QC) : Chenelière Education, 2006 